\title{
Characterisation of water-oil drainage process through a reservoir rock sample by digital core analysis
}

\section{Diego Casagrande*}

Dipartimento di Ingegneria,

Università degli Studi di Bergamo,

24044 Viale Marconi, 5, Dalmine (BG), Italy

Email: diego.casagrande@unibg.it

*Corresponding author

\section{Marzio Piller}

Dipartimento di Ingegneria e Architettura, Università degli Studi di Trieste,

34127 via A. Valerio 6/1, Trieste (TS), Italy

Email: piller@units.it

\section{Maurizio Santini}

Dipartimento di Ingegneria,

Università degli Studi di Bergamo,

24044 Viale Marconi, 5, Dalmine (BG), Italy

Email: maurizio.santini@unibg.it

\section{Gianni Schena}

Dipartimento di Ingegneria e Architettura, Università degli Studi di Trieste,

34127 via A. Valerio 6/1, Trieste (TS), Italy

Email: schena@units.it

\begin{abstract}
Oil-water relative permeability and capillary pressure of a water-wet digital core are computed using the finite-volume/volume of fluid method and mimicking traditional laboratory steady-state experiments carried out on a water saturated sample. Commercial software is used for immiscible multiphase fluid flow simulations, carried out on a low-cost multicore workstation, and the porescale data are upscaled to derive relative permeability and capillary pressure curves as function of water saturation. The digital sample is obtained by high resolution X-ray computed tomography scanning. [Received: March 13, 2013; Accepted: June 25, 2013]
\end{abstract}

Keywords: relative permeability; digital rock; pore-scale simulation; two-phase flow; steady-state; drainage process; water-oil system; volume of fluid. 
Reference to this paper should be made as follows: Casagrande, D., Piller, M., Santini, M. and Schena, G. (2014) 'Characterisation of water-oil drainage process through a reservoir rock sample by digital core analysis', Int. J. Oil, Gas and Coal Technology, Vol. 8, No. 4, pp.399-416.

Biographical notes: Diego Casagrande received his Laurea degree in Environmental Engineering from University of Trieste. He is currently a $\mathrm{PhD}$ student. His area of scientific interest is multiphase flow in porous media.

Marzio Piller received his $\mathrm{PhD}$ in Thermodynamics and Fluid Mechanics from the University of Trieste, Italy. He is an Assistant Professor at the Department of Engineering and Architecture, University of Trieste. His research interests are related to the numerical simulation of turbulent flows and flows through porous media.

Maurizio Santini is an Assistant Professor at the Department of Engineering, University of Bergamo, Italy. His research interests are related to the impact of liquid drops on solid surfaces and to the use of high-speed optical techniques.

Gianni Schena received his $\mathrm{PhD}$ in Engineering at the Imperial College, London. He is an Associate Professor at the Department of Engineering and Architecture of the University of Trieste, Italy. His research interests include high-resolution X-ray tomography and image analysis of reservoir rocks.

\section{Introduction}

Relative permeability and capillary pressure are petro-physical parameters fundamental for reservoir simulation and for devising exploitation scenarios. Two protocols are routinely used for experimental measurements. The first adopts a procedure where a single invading fluid displaces the defending fluid that initially saturates the pore space, recording the fluid production curves and the pressure drop through the sample after breakthrough (Loomis and Crowell, 1962). The recorded measurements require further processing to derive relative permeability and capillary pressure curves over a range of fluid saturation. In the jargon of petro-physics this experimental procedure is also referred to as unsteady state method (Akin, 2001; Toth et al., 2002). A history matching technique allows to derive relative permeability (and capillary pressure) values from a single experiment. A prescribed cost function is minimised to match the experimental data with those predicted by a two-phase core flow simulator designed to simulate special core analysis (SCAL) experiments (Loeve et al., 2011). This data processing technique is very sensitive to the boundary conditions chosen for the history match and does not assure the uniqueness of the solution (Tavassoli et al., 2005). The Johnson-Bossler-Neumann (JBN) method is sometimes used as an alternative to history match to derive relative permeability versus saturation profiles (Johnson et al., 1959). The JBN is derived from Buckley-Leverett's displacement theory (Buckley and Leverett, 1942) that does not comply fully with practical experiments, as it neglects capillary and gravitational forces and therefore assumes high flowrates. It provides relative permeability values only for saturations higher than breakthrough saturation. In addition the JBN assumes that the core sample is homogeneous. Alternatively to JBN that requires 
constant injection rate, Toth et al. (2002) have proposed a procedure that is more general for it may be applied also to constant pressure drop experiments.

The second experimental procedure requires injecting simultaneously the two fluids into a saturated sample, in a fixed volumetric ratio, and allows to find a pair of relative permeability values (one for each fluid) when stationary state and constant sample saturation are reached. Multiple experiments, each with different injection ratio, are necessary to estimate the entire relative permeability curve over a range of saturation. This experimental procedure is referred to as steady-state method even if the fluid-dynamics is typically non-stationary (Tiab and Donaldson, 2004).

In general the numerical methods available to study, characterise and synthesise via relative-permeability and capillary-pressure the dynamics of multiphase flow of immiscible fluids (i.e., oil and water or brine and super-critical $\mathrm{CO}_{2}$ ) in the pore-space of reservoir rock samples such as sandstone mimic routine laboratory measurements. Pore-scale fluid dynamic simulations are nowadays used to investigate pore-scale flow and transport processes in reservoir rocks (IJOGCT Special Issue, 2012). Zaretskiy et al. (2012) use the mixed finite-element/finite-volume model CSMP++ (Matthï et al., 2007) for simulating the pore-scale fluid flow and solute transport accompanied by chemical reactions in a digitalised Fontainebleau sandstone sample. Prodanović et al. (2012) use a commercial code from Itasca Consulting Group (PFC3D), originally developed by Cundall and Strack (1979), to study the movement of grains in a disordered package of spheres as it occurs in a gas drainage of a water saturated unconsolidated sediment. Dewers et al. (2012) study mudstone samples and use stacks of digital images acquired with focused ion beam-scanning electron microscopy (FIB-SEM) at $7.14 \mathrm{~nm}$ pixel size. Digital image analysis techniques (3DMA-rock software package, http://www.ams. sunysb.edu/ lindquis/3dma/3dma_rock/3dma_rock.html/) are used to determine pore morphology and pore size distribution. The absolute permeability of the samples is computed by pore-scale numerical simulations, using the commercial finite-element software COMSOL Multiphysics ${ }^{\circledR}$ (http://www.comsol.com/) and computational grids generated with $\operatorname{ScanIP}^{\circledR}\left(S_{\text {SanIP }}{ }^{\circledR}\right.$ by Simpleware, http://www.simpleware.com/ software/scanip/). The estimated permeabilities are in the range of 2 to 12 nanodarcies $(\mathrm{nD})$, in remarkably good agreement with experimental laboratory measurements on core plugs ( 5 to $50 \mathrm{nD})$.

Laboratory experiments require time-consuming and costly coring activities to recover lab-grade test samples (i.e., plugs) from the wellbore. At high borehole depths, as reached in nowadays oil wells, recovering rock plugs of suitable dimensions becomes more costly and time-consuming. The digital-lab workflow proposed here provides a fast and cheap routine for computing petro-physical properties from small samples such as drilling cutting or crushed sidewall when suitable cores are not available. Thus, the suggested procedure is complementing and corroborating traditional experimental laboratory data, the integration of petro-physical data at various scale remaining an important issue. Obviously, the influence of the rock heterogeneities are captured at the micro-meter scale and cannot provide all the information carried of a full diameter core (e.g., systems of fractures and their orientation). Here the finite-volume/volume-of fluid (FV-VOF) commercial solver FLUENT ${ }^{\circledR}$ (FLUENT ${ }^{\circledR}$ Theory Guide, http://www.ansys. $\mathrm{com} /$ ) is used for the simulation of the oil/water flow in the pore-space of a sandstone rock that has been reconstructed using high resolution X-ray computed tomography (Coles et al., 1998; Josh et al., 2012) and segmented by image processing tools. For 
relative permeability calculation the steady-state experiment is reproduced numerically. This requires a set of numerical experiments in contrast to the single experiment sufficient for the unsteady method. Notwithstanding the higher computational effort, the choice is dictated by the will to avoid uncertainties related to the history match technique. Also the capillary pressure $\left(P_{c}\right)$ curve is computed monitoring the steady state experiments and recording the difference in pressure between the non-wetting and the wetting phase. The Ansys-FLUENT ${ }^{\circledR}$ finite volume model has been used previously by Crandall et al. (2012a, 2012b) to simulate at pore-scale the behaviour of supercritical- $\mathrm{CO}_{2} /$ brine systems in a sandstone sample, reconstructed by X-ray $\mathrm{CT}$, under the simplifying assumption of fluid immiscibility.

The following issues are covered by the paper:

1 The FV-VOF computational method is briefly recalled as the mesoscopic immiscible lattice Boltzmann model (ILBM) by Gunstensen et al. (1991), which is probably the most widely used LBM for simulating two phase (i.e., oil/water) flow in porous media reconstructed with micro tomography (Arns et al., 2004; Papafotiou et al., 2008).

2 The relative permeability and the capillary pressure curves obtained by the proposed methodology are presented.

3 Three methods for estimating the threshold capillary pressure are presented.

\section{Experimental and numerical methods}

\section{$2.1 \quad$ X-ray tomography}

X-ray computed tomography (XCT) has been used for long time in petrophysical laboratories for pore-space visualisation and for related post processing including morphological measurements (Coles et al., 1998; Josh et al., 2012). More recently it has been used to visualise the occupancy of immiscible fluids in saturated pore spaces, in general by doping one of the fluids to improve their density contrast. For example one can discriminate between oil and water when an iodine-based dopant is used (Iglauer et al., 2010). More recently, X-ray tomography has been used to produce digital rockmodels, suitable for micro-scale simulation (Hazlett et al., 1998; Nguyen et al., 2006; Piller et al., 2009).

Millimetric samples of porous rocks such as drilling cuttings are semitransparent to hard X-ray (energies from about 12 to $120 \mathrm{keV}$ ) and digital radiographies with micrometric planar resolution can be taken. The rockpore density contrast is favourable to planar grey scale images with high dynamic range. By taking radiographies from different viewpoints and applying tomography principles, the internal structure of the porous rock can be reconstructed. For detectors with square pixels, the cubic voxel side of the reconstructed tomographic volume is related to the geometric magnification of the tomographic system. The true planar resolution of the projection images is invariably degraded by the interpolation that the backprojection algorithms apply by redistributing the detector pixel luminosity to all the voxels laying on the path of the X-ray between the focal-spot and the pixel. This results in a true 3D tomographic resolution lower than the nominal. However, micrometric or even sub-micrometric spatial resolutions once 
possible only at synchrotron beam-lines now are attainable also with laboratory systems, such as the one shown in Figure 1.

Figure 1 One-micron focal spot cone-bean source of the X-ray computed tomography system at University of Bergamo

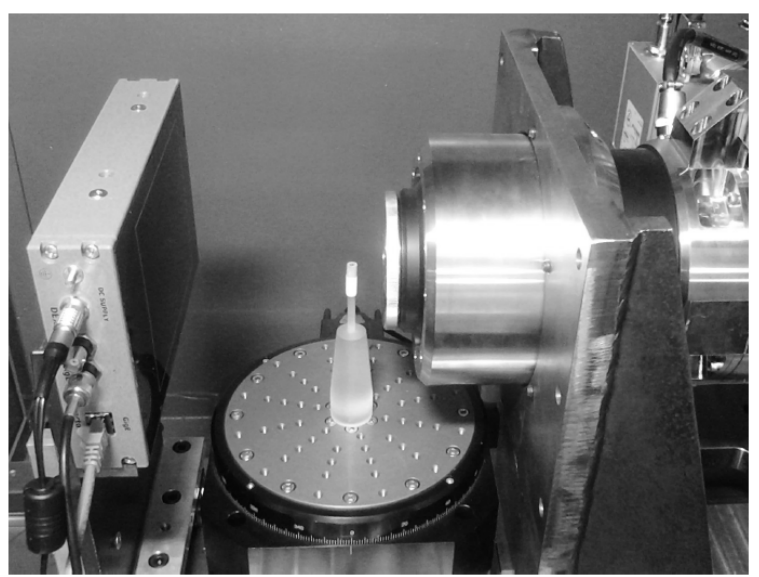

Typical scanning time is one hour, for micro-focus cone-beam-based laboratory machines in applications requiring micrometric nominal resolution and $2,000^{3}$ voxels. Tomographic beam-lines at high X-ray photon-flux synchrotrons allow scanning and registration of log-processed radiographs in less than ten minutes.

In this work a sandstone sample is used. The voxel size is $4.5 \mu \mathrm{m}$ and the size of the considered digital sample, shown in Figure 2, is $200^{3}$ voxels. The porosity is $11.4 \%$. The experimental absolute permeability of the rock from which the small plug was extracted is about $200 \mathrm{mD}$.

Figure 2 Volume rendering of the sandstone sample (see online version for colours)

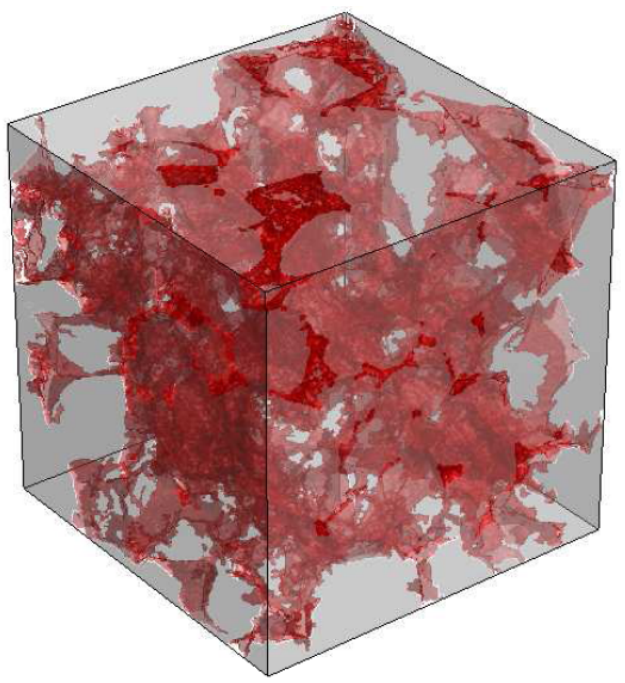

Notes: The pore-grain interface is shown in red. The rock matrix is coloured in grey. 


\subsection{Volume of fluid}

Among the computational fluid dynamic (CFD) methods currently available for the simulation of multiphase flow in porous rocks, the ILBM have emerged rapidly (Succi, 2001; Wolfram, 1986). The elemental fluid cell is identified with the (cubic) voxel of the wet pore-space as segmented from the tomographic volume. Thus, explicit meshing of the pore-space is not necessary. The wet cells of this regular 3D set are populated by particles that can move (i.e., stream) according to 18 discrete velocities or remain at rest when the most common D3Q19 lattice geometry is adopted. Within one cell the microscopic particle population is distributed according to a particle-distribution-function that evolves in time. Cell macroscopic physical properties and hydrodynamic variables can be computed by microscopic lattice population-fractions. The Bhatnagar-GrossKrook collision model (Bhatnagar et al., 1954) with an equilibrium distribution function allows time evolution. Particle streaming allows space evolution. Details on the time-space discretisation of the Boltzmann equation are available in many general reference texts (Succi, 2001). For two phase incompressible flows two particle distributions are necessary at each wet cell site thus doubling the amount of data with respect to the single phase case. The dynamics of the fluid-fluid interface are governed by additional forcing terms that mimic the interaction between immiscible fluids. A strategy to keep a thin interface limiting the smearing is also needed. Fluid wettability is introduced by adding a fluid-rock adhesive term rather than a contact angle. LBM requires well resolved ducts to properly develop fluid flow advection and interfaces identification. The problem might be tackled by resizing the volume image according to a magnification factor, accepting the considerable computational overburden.

The existence of large flow paths is verified with a path extraction method based on the scalar field generated with a weighted geodesic distance transform of the pore space. The weights are generated with a Euclidian distance transform applied to the pore space. Details are given elsewhere (Schena and Favretto, 2007).

An alternative to LBM is to resort to traditional CFD methods as the FV-VOF implemented in the commercial solver FLUENT ${ }^{\circledR}$. The VOF is a technique widely used to tackle problems in which two or more fluids are present and the dynamics of the interface is of interest. It was first introduced by Nichols and Hirt $(1975,1981)$. Various improvements have been developed subsequently and a non-exhaustive list is reported by Tang et al. (2004). Here, VOF is used for tracking the shape of the water/oil interface. The phase volume fraction is defined at each computational cell of the fixed regular and uniform computational grid adopted. The volume fraction function is discontinuous passing through the interface. The piece-wise linear interpolation reconstruction (PLIC) algorithm is used in the present work for interface tracking. The method is coupled with the continuum surface force (CSF) algorithm for surface force modelling that adds a source term in the momentum equation that is proportional to the interface curvature (Brackbill et al., 1992). An excellent review of the VOF-PLIC steps for interface tracking and multiphase fluid advection is given by Tang et al. (2004).

The pressure-implicit with splitting of operators (PISO) scheme for pressure-velocity coupling is used (Ferziger and Perić, 2001). The non-iterative time advancement (NITA) option for time stepping has been preferred in the present calculations for computing efficiency since it reduces the computational time and allows performing only one outer iteration per single time step. 


\subsection{Computational domain and mesh}

Each voxel of the segmented pore space volume corresponds to a cubicmesh element. An alternative approach consists in generating a triangulation for the pore-grain interface, identified by a contouring algorithm; an unstructured (in general tetrahedral) volumemesh is then built upon the surfacemesh. The cubicmesh can be generated much faster and, in addition, it does not require the use of sophisticated and costly mesh generation packages (Gerbaux et al., 2010). The resulting pore/rock surface is stair-step like for the cubic mesh and smooth for the tetrahedral mesh. This feature could be used to criticise the cubic mesh approach. However, one should recognise that the base geometry, provided by the XCT, is voxel-based and any smoothing operation is necessarily arbitrary: the stair-step boundary of the cubic mesh represents the surface roughness elements of dimension comparable to the voxel side. The pore-grain interface can be identified by the list of linear indexes of the voxels lying on the border of the pore space and connected to grains according to a given voxel-neighbourhood connectivity. For fluid dynamic simulation a connectivity of 6 must be used, as fluxes propagate across the control volume faces. Given a connectivity of 6 , one voxel of the pore space is on the pore-grain interface if at least one of the six (connected) neighbours is part of a solid grain. The identification of the interface fluid voxels is required for subsequent selection of the boundary surface where the boundary conditions are enforced. The computational domain and the related computational mesh are trivially generated by associating each voxel to a hexahedral computational cell. Even for large volumes, the mesh generation process takes just a few minutes on a desktop computer. Even though the identification of voxels with computational cells allows an easy mesh generation process, some regions might be under-resolved. The most natural way to overcome this problem is to use one of the many mesh adaptivity capabilities provided by most commercial CFD packages. The reported results have been obtained using the hanging nodemesh adaptation technique available with FLUENT $^{\circledR}$. The base mesh used in the present simulations contains about $9.80 \times 10^{5}$ cells.

The problem of identifying suitable dimensions for the representative elementary volume (REV), as defined, among others, by Bear (1972), is not addressed in the present study. A modern interpretation of the REV relies on the availability of a sufficient number of samples, in order to define representative statistical moments (Hilfer, 2002; Kanita et al., 2003).

FV-VOF solvers are computationally intensive but in general less amenable to parallelisation than LBM. CFD frequently ran on high power computing (HPC) facilities. The communication between processors is often the bottleneck. Here this problem has been resolved by running our simulations on a new generation top performance eight-core processors. Tomographic volumes of size say $400^{3}$ can be processed on the desktop machine in tens of hours. On a dual socket workstation even larger volumes can be processed in the same computational time and permeabilities corresponding to different saturation calculated at the same time.

\section{Results and discussion}

As previously mentioned, in our study we co-inject oil and water in a waterwet sample. The considered fluid properties are as follows: 


$$
\begin{aligned}
& \rho_{w}=998.2 \mathrm{~kg} / \mathrm{m}^{3} ; \mu_{w}=0.001 \mathrm{~kg} / \mathrm{ms} \\
& \rho_{o}=800.0 \mathrm{~kg} / \mathrm{m}^{3} ; \mu_{o}=0.003 \mathrm{~kg} / \mathrm{ms} \\
& \sigma_{w, o}=0.05 \mathrm{~N} / \mathrm{m} ; \theta=30^{\circ}
\end{aligned}
$$

where $\delta$ and $\mu$ are the density and dynamic viscosity of the fluids, $\sigma_{w, o}$ is the surface tension and $\theta$ is the contact angle.

The water layer covers the pore surface, while the oil fills the core of the pore body. Thus at the fluid inlet section of the meshed pore-space, specialised image-processing tools are used to identify the boundaries of the pores and to adjust the thickness of the water layer according to the prescribed water/oil volumetric ratio.

The simulations are carried out for different values of the volumetric fluid ratio and with an entry velocity $(0.01 \mathrm{~m} / \mathrm{s})$ yielding a pore-scale Reynolds number, based on the square root of the absolute permeability, of $4 \times 10^{-3}$. Numerical methods allow for measurements on internal sections of the sample that are not possible with traditional laboratory experiments which provide results with respect to inlet and outlet sections only. Nevertheless considerably larger volumes of rock may be flooded in the experiments. The output data are then upscaled to estimate relative permeability and capillary pressure versus saturation, that are the set of data necessary for macro-scale reservoir simulation.

\subsection{Relative permeability}

In the procedure used here, the two fluids invade the digital core sample, initially fully saturated with water, until the produced fluid ratio stabilises. The analysed process is a drainage. At the final saturation, the effective permeability to each phase is obtained by using Darcy's law:

$$
\begin{aligned}
& q_{w}=-\frac{k k_{r, w}}{\mu_{w}} \nabla p_{w} \\
& q_{o}=-\frac{k k_{r, o}}{\mu_{o}} \nabla p_{o}
\end{aligned}
$$

where $q_{w, o}$ denotes the specific discharge of the considered phase, $k$ and $k_{r}$ are the absolute and relative permeabilities, respectively and $p_{w, o}$ is the phase pressure. Numerically, both the constant injection rate and the constant pressure drop cases can be simulated. The sample average saturation, pressure drop and flow rates are continuously monitored during the transient process. The distribution of the two fluid phases at an advanced stage of drainage is shown in Figure 3, where the sandstone material is not shown. The oil phase is shown in red. The water phase is shown in transparent blue. Velocity inlet and pressure outlet boundary conditions are used to drive the injection. Inlet velocity is $0.01 \mathrm{~m} / \mathrm{s}$. The volume rendering shows the complexity of the process with differential advancement of menisci inside the porous space. The advancement and the evolution of the fluid interfaces and their morphology is continuously visualised. This is of great help for the full understanding of the fundamental behaviours of these pore-scale non-equilibrium processes. Fingering instability of the displacing front due to the randomness of the pore length scales, viscous fingering, snap-off, bubble clustering growth, ganglia formation and coalescence are some pore-scale dynamic phenomena that 
can be captured. Trapping mechanisms and the related residual saturations of the non-wetting phase (and the irreducible saturation of the wetting one) are associated to these complex interfacial phenomena, the media topology and the rock wettability.

Figure 3 Visualisation of the evolution of the oil phase (shown in red) during a drainage process in a 2003 voxels sample (see online version for colours)

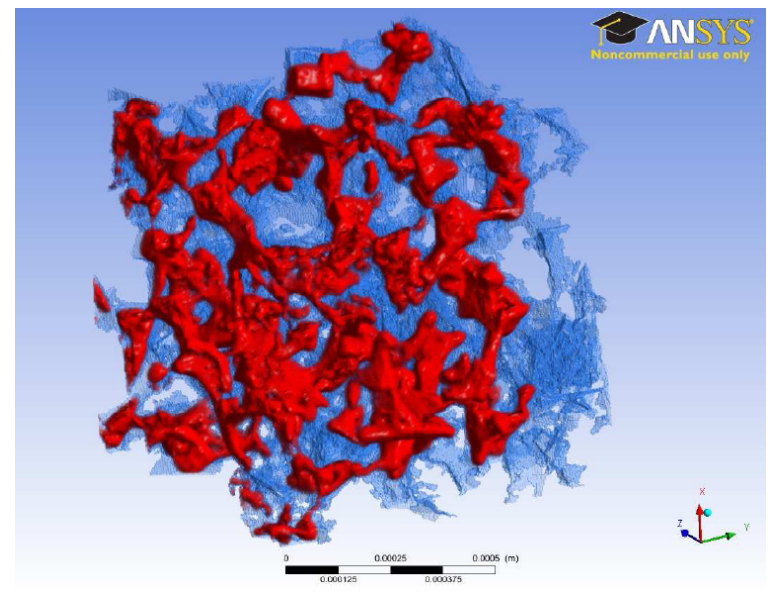

Note: The pore-grain interface is shown in light blue.

The absolute permeability is computed with a mono-phase numerical experiment with the method described by Piller et al. (2009). The fluid injection velocity significantly influences the computed values. The absolute permeability of the sample has been estimated injecting oil and subsequently recomputed injecting water. At high rates (e.g., above $0.1 \mathrm{~m} / \mathrm{s}$ ) the pore Reynolds number does not assure the validity of Darcy's law and the absolute permeability of the two fluids are sensibly different. The permeability values converge to the same value only at lower rates (e.g., $0.01 \mathrm{~m} / \mathrm{s}$ ). This velocity value has been adopted as inlet velocity at the injection section. The computed relative permeability curves are presented in Figure 4(a) and fitted with the classical two-parameter Corey correlation (Brooks and Corey, 1996):

$$
\begin{aligned}
& k_{r, w}=k_{r, w, \max }\left(\frac{S_{w}-S_{w, i}}{1-S_{w, i}-S_{o, r}}\right)^{n_{w}} \\
& k_{r, o}=k_{r, o, \max }\left(\frac{1-S_{w}-S_{o, r}}{1-S_{w, i}-S_{o, r}}\right)^{n_{o}}
\end{aligned}
$$

The irreducible water saturation $S_{w, i}$ is computed by extrapolation with piece-wise cubic Hermite polynomial interpolation (PCHIP) from the $k_{r, w}$ set of data prescribing $k_{r, w}\left(S_{w, i}\right)=0$. A similar PCHIP procedure allows to compute the residual oil saturation $S_{o, r}$ from the $K_{r, o}$ set by prescribing $K_{r, o}\left(S_{o, r}\right)=0$. The computed values are:

$$
S_{w, i}=0.1993 ; S_{o, r}=0.1791
$$

Each couple of points, at a fixed water saturation value, represents a single test. The comparison between the simulated and the experimental data is shown in Figure 4(b). 
Figure 4 Relative permeability as a function of the sample water saturation, (a) Computed data ( $\circ$ symbol), interpolating polynomial (solid line) and Corey fitting (dashed line) (b) Experimental data ( $\Delta$ symbols) and interpolating polynomial (solid line) for computed data ( $\mathrm{s}$ symbol) (see online version for colours)

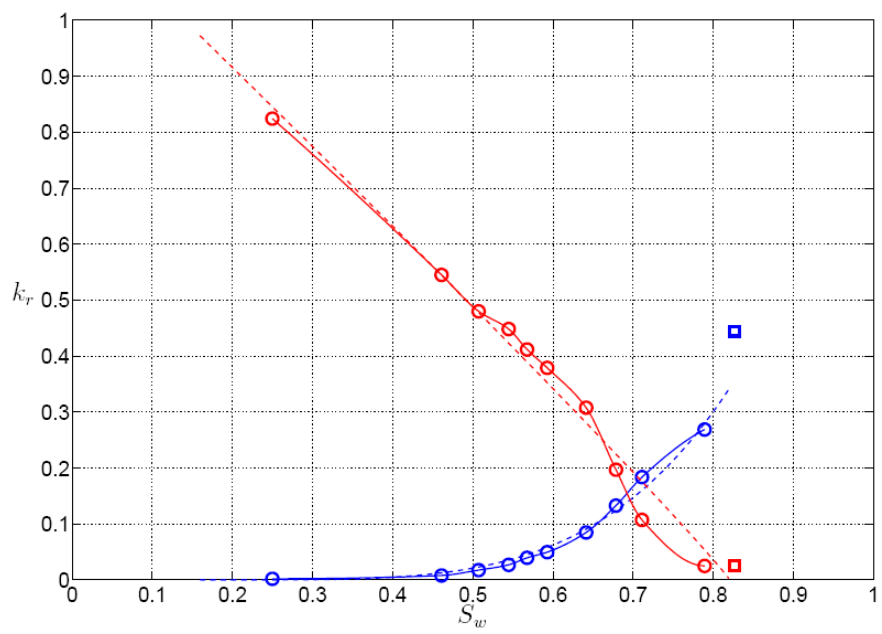

(a)

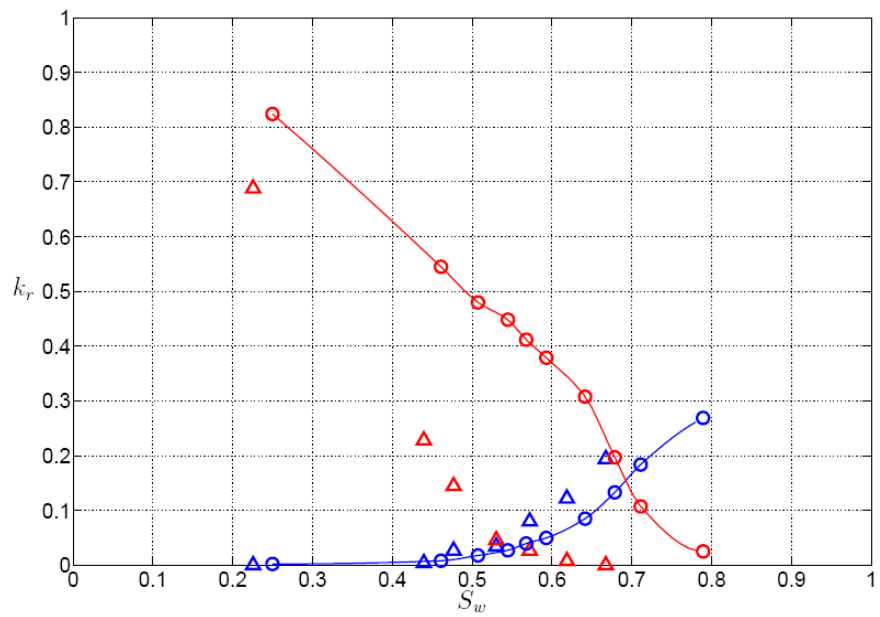

(b)

Note: Values of $k_{r}$ corresponding to $\square$ symbol are derived by an imbibition experiment.

The numerical method allows computing values across the entire saturation range. The calculated relative permeability curve for water is in good agreement with experimental data while a remarkable disagreement is observed for the oil relative permeability [Figure 4(b)]. The small dimensions of the digital sample might explain this discrepancy. The regression of the calculated relative permeabilities with the correlation by Brooks and Corey (1996) yields the following values for the parameters, appearing in equation (2): 


$$
\begin{aligned}
& k_{r, w, \max }=0.344 ; n_{w}=4.205 \\
& k_{r, o, \max }=0.972 ; n_{o}=0.953
\end{aligned}
$$

\subsection{Capillary pressure}

The capillary pressure $\left(P_{c}\right)$ represents a key parameter for reservoir studies. Because of the small size of the digital sample, volume-averaged pressures of the phases are computed at every time step and their difference yields the capillary pressure. Both pressure drop across the sample versus time and saturation versus time profiles, shown in Figure 5, are used to identify the attainment of stationary conditions necessary for measurements. One measurement at stationary state is a point of the capillary pressure curve. The computed capillary pressure data are fitted with the two-parameters Bentsen-Anlie function (Bentsen and Anlie, 1977) [see Figure 6(a)]:

$$
P_{c}=P_{c, t}-P_{c, s} \log \left(\frac{S_{w}-S_{w, i}}{1-S_{w, i}}\right)
$$

where $P_{c, t}$ is the threshold capillary pressure and $P_{c, s}$ is the so-called span pressure (Bentsen and Anlie, 1977). Comparison with experimental data is reported in Figure 6(b). The computed capillary pressure is in agreement with the experimental data for water saturations above $46 \%$. The regression of the calculated capillary pressure with the correlation by Bentsen and Anlie (1977) yields the following values for the parameters, appearing in equation (4):

$$
P_{c, t}=0.52 ; \quad P_{c, s}=0.88
$$

Figure 6 shows that different ranges of $S_{w}$ are covered by laboratory experiments (square symbol) and numerical experiments (star symbol). In laboratory experiments one fluid displaces another in imbibition or drainage processes, that are often carried out at displacement rates much higher than those typical of reservoir phenomena. The numerical methods allow simulating fluid flow displacement processes at lower velocities and prescribing a pore-scale Reynolds number (0.004 in the present simulations) thus ensuring laminar flow (see also Section 3 results and discussion). This results in the possibility to explore lower values of $P_{c}$ at high $S_{w}$ [Figure 6(b)].

While the injection oil/water ratio is imposed the resulting pore-space saturation is a dependent variable that is also influenced by the morphology of the pore space and its wettability. With a water fraction at the inlet section of $2 \%(8 \%)$ the final water saturation obtained is $46 \%(50 \%)$. With a drainage experiment with only oil injected the water saturation is $25 \%$. Thus, a very small increase in water fraction $(2 \%)$ prevents the oil to displace an additional $21 \%$ in water. This behaviour is shown as a saturation gap in Figures 6(a) and 6(b).

The threshold capillary pressure is the pressure at which the drainage process begins, in other words the pressure that should be exceeded before any oil can start flowing through the sample (Christiansen, 2008; Egerman et al., 2006). Here three alternative methods are used to estimate the $P_{c, t}$.

The first method is based on a morphological analysis of the pore-space. The largest pore radius in a 100 micron thick inlet section is estimated computing the medial path for passing through the thick section and inflating the maximal spheres with centre on 
the path. A method for generating the medial path that is used in virtual endoscopy is adopted (Schena and Favretto, 2007). The $P_{c, t}$ is then obtained from the well-known Young-Laplace equation:

$$
P_{c}=2 \frac{\sigma \cos (\theta)}{R}
$$

where $\sigma$ represents the interfacial tension between oil and water, $R$ is the radius of the largest maximum sphere with centre on the media axis, $\theta$ is the contact angle between the tangent to the interface and the solid surface. $R$ is estimated via distance transform. The $P_{c, t}$ value is 0.56 psi.

Figure 5 (a) Oil saturation versus simulation time, inlet water fraction $\alpha_{w}=8.81 \%$

(b) Pressure drop versus simulation time, inlet water fraction $\alpha_{w}=8.81 \%$

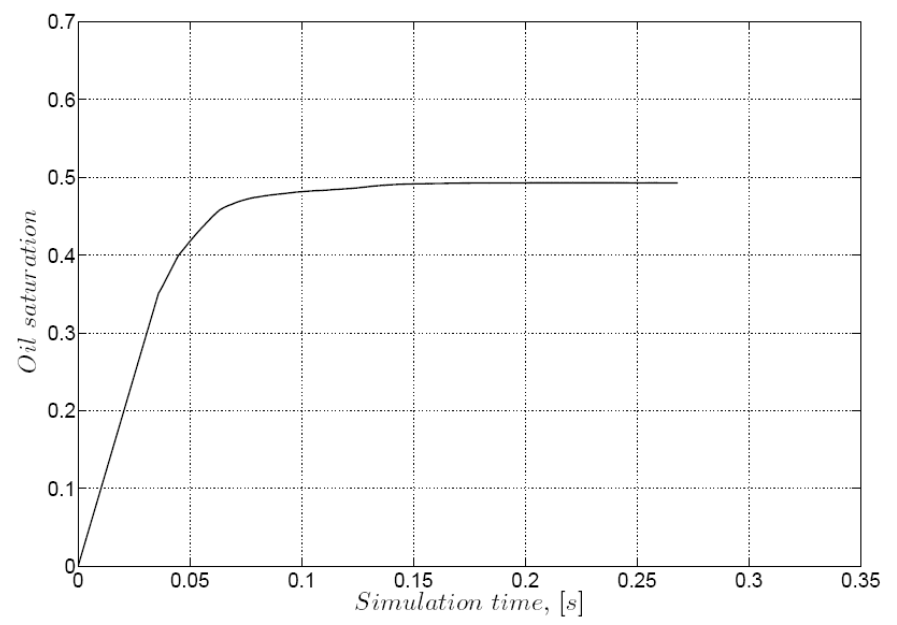

(a)

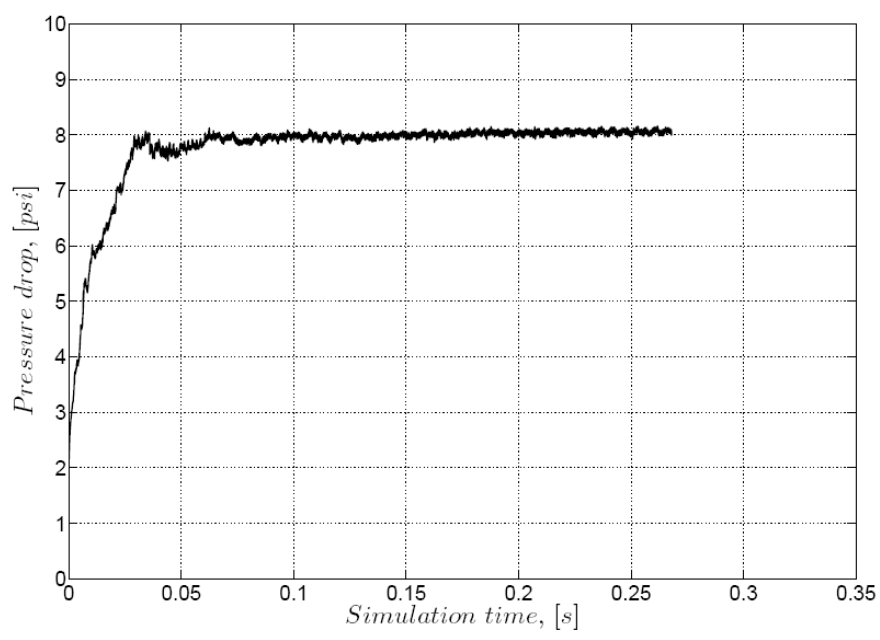

(b) 
Figure 6 Capillary pressure as a function of the sample water saturation, (a) Computed data and interpolating polynomial ( $\mathrm{s}$ symbol and solid line) and Bensten-Anli fitting (dashed line) (b) Experimental data ( $\Delta$ symbol) and interpolating polynomial (solid line) of the computed data ( $\circ$ symbol)

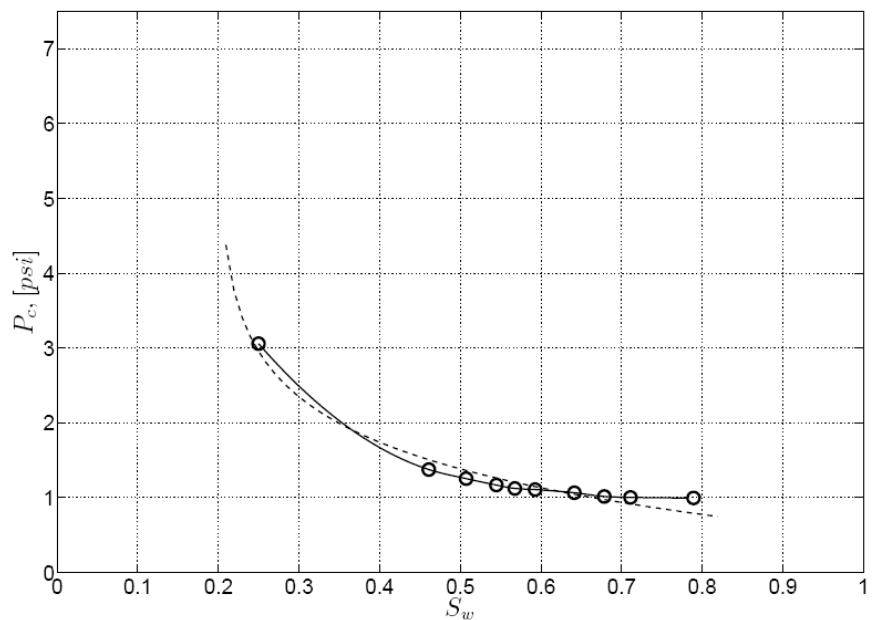

(a)

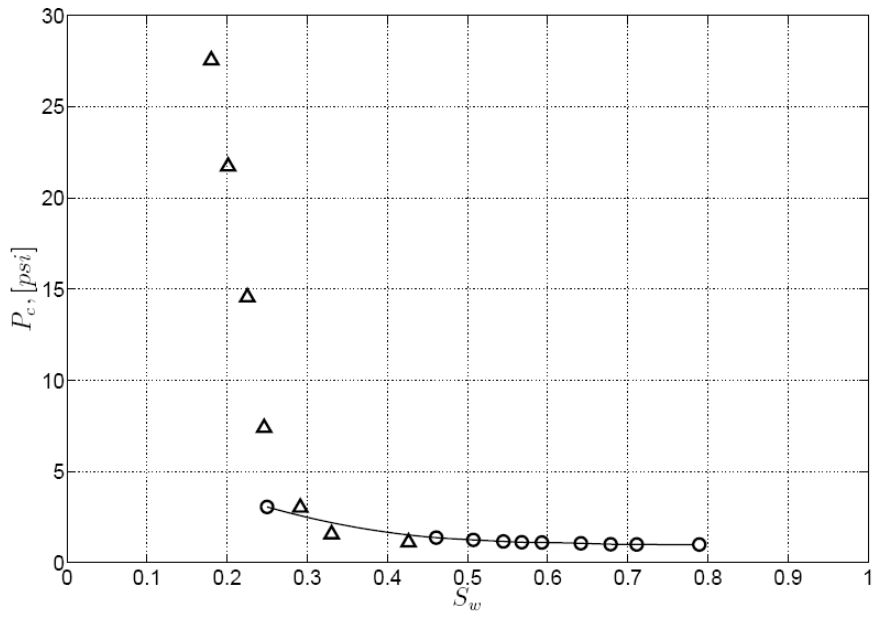

(b)

The second method for estimating $P_{c, t}$ exploits a displacement simulation that fulfils a classical protocol used for laboratory experiments (Christiansen, 2008). The core sample, saturated by water, is invaded by oil at very low flow rate $\left(2 \times 10^{-3} \mathrm{~m} / \mathrm{s}\right)$, in order to consider the pressure difference across the sample nearly equal to the capillary pressure. Instead, at higher injection rates, viscous resistance would increasingly dominate the pressure drop (Christiansen, 2008). The $P_{c, t}$ is measured at the very first stage of the injection process, as shown in Figure 7. The $P_{c, t}$ value is 0.34 psi. 
Figure 7 Capillary pressure as a function of oil saturation

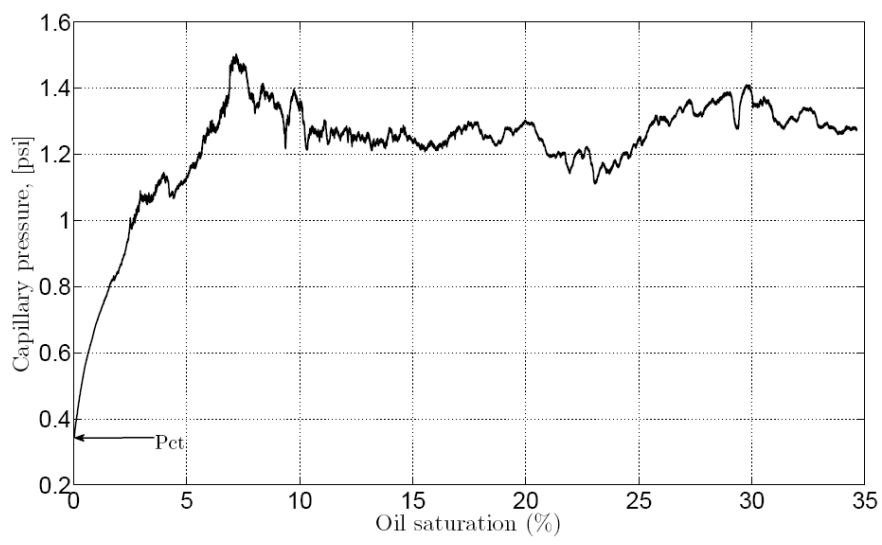

Note: Oil injection rate $0.002 \mathrm{~m} / \mathrm{s}$.

Figure 8 Capillary pressure as a function of the oil inlet velocity

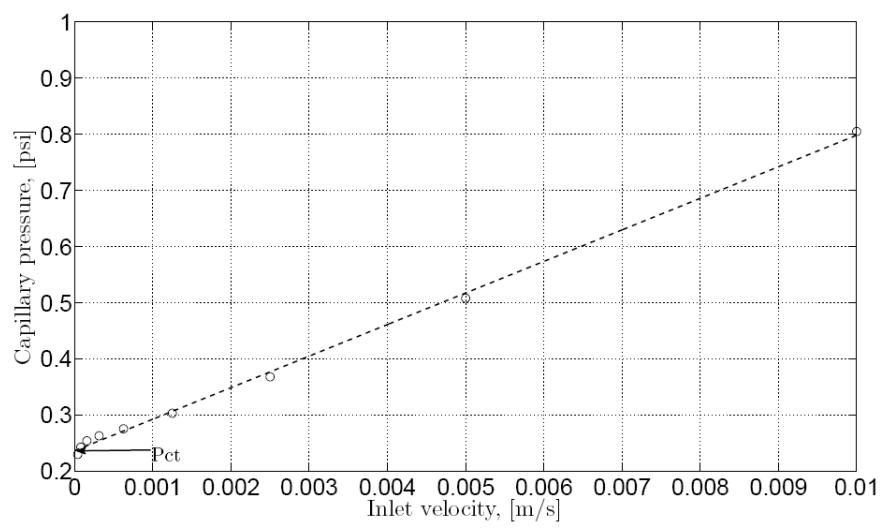

The third method for estimating $P_{c, t}$ consists in a step-by-step drainage. Oil is injected into the sample for a fixed number of simulation steps with a predefined injection rate. Then the simulation is repeated several times each time halving the oil rate. Finally, the threshold capillary pressure is derived as the intercept of the regression line fitting the capillary pressure versus velocity inlet, as shown in Figure 8 . The estimated $P_{c, t}$ is 0.24 psi. The step-by-step drainage is intended to compute a lower limit for the $P_{c, t}$. Namely, the method yields the minimum pressure difference, capable of intruding a minimum amount of oil through the inlet section of the sample. This pressure difference is therefore related to the capillary pressure acting across the largest opening on the inlet section. Nevertheless, the actual threshold capillary pressure corresponds to the pressure difference that has to be applied across the sample, to allow for a continuous filament of oil passing through the sample (Egerman et al., 2006). This pressure difference is related, in turn, to the capillary pressure acting across the largest among the smallest throats encountered along all the possible paths connecting the inlet and outlet sections of the sample. These considerations provide an explanation for the lowest value of $P_{c, t}$ returned by the third method, among all the proposed methods and suggest that the third method 
should yield reliable results when applied to a sufficiently large sample, such that the areal pore-size distribution does not differ significantly from the volumetric pore-size distribution. In spite of the aforementioned limitations, the method has the lowest computational cost among the proposed methods for the calculation of $P_{c, t}$ and, for the present sample, yields a value of $P_{c, t}$ of the same order of magnitude as the other methods.

The graph in Figure 7 shows unstable values of capillary pressure at increasing values of saturation. It refers to a capillarity pressure experiment carried out according to the aforementioned method proposed by Christiansen (2008), exclusively devoted to threshold capillary pressure estimation. The experiment is carried out with injection velocity $0.002 \mathrm{~m} / \mathrm{s}$, i.e., 50 times lower than that used for capillary pressure and relative permeability estimations. In addition these measurements of capillarity pressure are taken before breakthrough and therefore well away from the plateau where the saturation reaches the stability. In general, minor capillary pressure instability can manifest also at stable values of saturation since it is related to the change in morphology of the oil/water interface.

Table 1 Threshold capillary pressure calculated with different methods

\begin{tabular}{lc}
\hline Method & $P_{c, t}[p s i]$ \\
\hline Morphological method & 0.56 \\
Slow drainage $v_{\text {in }}=0.002 \mathrm{~m} / \mathrm{s}$ & 0.34 \\
Step-by-step drainage & 0.24 \\
Fitting, equation (4) & 0.52 \\
\hline
\end{tabular}

\section{Concluding remarks}

Nowadays a few companies offer digital rock physics services and have proprietary code based on the Rothman-Gunstensen (Gunstensen et al., 1991) lattice Boltzmann model. The use of commercial CFD software to compute the relative permeability and the capillary pressure paves the way to the diffusion of numerical methods for petro-physical computing, to the standardisation of the digital rock workflow and to the reproducibility of numerical results. In this paper, we use ANSYS-Fluent ${ }^{\circledR}$ that is a very well-established commercial product with a large basis of users. A large number of benchmarks are published in the literature showing the adherence between laboratory and Fluent-simulated multiphase experiments. However a number of free open source CFD packages exists for multiphase simulation (e.g., OpenFoam User Guide, http://www. openfoam.com/). In general, the reliability of the entire digital rock physics workflow is founded on the use of a REV. This issue is not examined in this paper where a REV sub-volume is processed. However one can foresee that larger tomographic volumes (e.g., $1,000^{3}$ voxels or more) are amenable to be processed provided that adequate computational resources are available. Furthermore, a compromise between a resolution sufficient to capture the narrowest throat giving contribution to flow and a digital volume amenable to be processed with the computing resources available is required.

The CFD procedure adopted here models the physical process in which one or two immiscible fluids are co-injected and displace the fluid/s that initially occupy the pore space. The procedure uses the full morphology of the rock as reconstructed by X-ray CT. 
According to the available literature and to the authors' best knowledge, the use of a traditional CFD model for two-phase flow simulations at pore scale is an original contribution of the present work. Relative permeability and capillary pressure curves are calculated according to the so called steady-state experiment. The relative permeability of each phase is computed via Darcy law where the oil pressure drop and the water pressure-drop are computed separately rather than using the mixture pressure drop. The threshold capillary pressure is computed following three different and independent strategies that yield comparable results and also in line with samples of similar permeability (Bentsen and Anlie, 1977).

\section{Acknowledgements}

The financial support from Università degli Studi di Trieste - Fondo Ricerca Ateneo (FRA 2012) is gratefully acknowledged.

\section{References}

3DMA-rock software package, A Software Package for Automated Analysis of Rock Pore Structure in 3-D Computed Microtomography Images [online] http://www.ams.sunysb. edu/ lindquis/3dma/3dma_rock/3dma_rock.html (accessed 28 May 2013).

Akin, S. (2001) 'Estimation of fracture relative permeabilities from unsteady state corefloods', $J$. Petrol. Sci. Eng., Vol. 30, No. 1, pp.1-14.

Arns, C.H., Knackstedt, M.A., Val Pinczewski, W. and Martys, N.S. (2004) 'Virtual permeametry on microtomographic images', J. Petrol. Sci. Eng., Vol. 45, pp.41-46.

Bear, J. (1972) Dynamics of Fluids in Porous Media, Dover Publications, New York, USA.

Bentsen, R.G. and Anlie, J. (1977) 'Using parameter estimation techniques to convert centrifuge data into a capillary pressure curve', Trans. AIME, Vol. 17, No. 1, pp.57-64.

Bhatnagar, P.L., Gross, E.P. and Krook, M. (1954) 'A model for collision processes in gases. I. Small amplitude processes in charged and neutral one-component systems', Physical Review, Vol. 94, No. 3, pp.511-525.

Brackbill, J.U., Kothe, D.B. and Zemach, C. (1992) 'A continuum method for modeling surface tension', J. Comp. Phys., Vol. 100, No. 2, pp.335-354.

Brooks, R. and Corey, A.T. (1996) 'Properties of porous media affecting fluid flow', Journal of the Irrigation and Drainage Division, Proceedings of the American Society of Civil Engineers, Vol. 92, No. IR2, pp.61-88.

Buckley, S.E. and Leverett, M.C. (1942) 'Mechanism of fluid displacement in sands', Trans. AIME, Vol. 146, No. 1, pp.107-116.

Christiansen, R.L. (2008) Multiphase Flow through Porous Media, KNQ Engineering Publishers, Salt Lake City, Utah.

Coles, M.E., Hazlett, R.D., Spanne, P., Soll, W.E., Muegge, E.L. and Jones, K.W. (1998) 'Pore level imaging of fluid transport using synchrotron X-ray microtomography', J. Pet. Sci. Tech., Vol. 19, Nos. 1-2, pp.55-63.

COMSOL Multiphysics ${ }^{\circledR}$ [online] http://www.comsol.com/ (accessed on 29 May 2013).

Crandall, D., Warzinski, R.P. and O’Connor, W.K. (2012a) 'Examining how $\mathrm{CO}_{2}$ displaces brine at the pore level', Proceedings of the International Society of Porous Media 2012 Annual Meeting, West Lafayette IN, May 2012. 
Crandall, D., Warzinski, R.P., O’Connor, W.K., Kabir, A.M. and Bromhal, G. (2012b) 'Pore scale $\mathrm{CO}_{2}$ displacement in sandstone with comparison to core scale dynamics', Proceedings of the 12th AIChE Annual Meeting, Pittsburgh, PA, November 2012.

Cundall, P.A. and Strack, O.D.L. (1979) 'Discrete numerical model for granular assemblies', Geotechnique, Vol. 29, No. 1, pp.47-65.

Dewers, T.A., Heath, J., Ewy, R. and Duranti, L. (2012) 'Three-dimensional pore networks and transport properties of a shale gas formation determined from focused ion beam serial imaging', International Journal of Oil, Gas and Coal Technology, Vol. 5, Nos. 2-3, pp.229-248

Egerman, P., Lombard, J.M. and Bretonnier, P. (2006) 'A fast and accurate method to measure threshold capillary pressure of caprocks under representative conditions', SCA Paper A46 prepared for International Symposium of the Society of Core Analysts, Trondheim, Norway, September.

Ferziger, J.H. and Perić, M. (2001) Computational Methods for Fluid Dynamics, Springer, Berlin.

FLUENT $^{\circledR}$ Theory Guide [online] http://www.ansys.com (accessed 24 May 2013).

Gerbaux, O., Buyens, F., Mourzenko, V.V., Memponteil, A., Vabre, A., Thovert, J-F. and Adler, P.M. (2010) 'Transport properties of real metallic foams', Journal of Colloid and Interface Science, Vol. 342, No. 1, pp.155-165.

Gunstensen, A., Rothman, D.H., Zaleski, S. and Zanetti, G. (1991) 'A lattice-Boltzmann model of immiscible fluids', Physical Review A, Vol. 43, No. 8, pp.4320-4327

Hazlett, R.D., Chen, S.Y. and Soll, W.E. (1998) 'Wettability and rate effects on immiscible displacement: lattice Boltzmann simulation in microtomographic images of reservoir rocks', J. Petrol. Sci. Eng., Vol. 20, Nos. 3-4, pp.167-175.

Hilfer, R. (2002) 'Review on scale dependent characterization of the microstructure of porous media', Transp. Porous Media, Vol. 46, Nos. 2-3, pp.373-390.

Iglauer, S., Favretto, S., Spinelli, G., Schena, G. and Blunt, M.J. (2010) 'X-ray tomography measurements of power-law cluster size distributions for the nonwetting phase in sandstones', Phys. Rev. E, Vol. 82, No. 5, pp.056315-056317.

IJOGCT Special Issue (2012) 'Special issue on pore-scale flow and transport processesin petroleum reservoirs', International Journal of Oil, Gas and Coal Technology, Vol. 5, Nos. 2-3, pp.142-156, Guest Editor Professor Karpyn, Z.T. [online] http://www.inderscience.com/ info/inarticletoc.php? jcode=ijogct\&year $=2012 \&$ vol $=5 \&$ issue $=2 / 3$ (accessed 26 July 2013).

Johnson, E.F., Bossler, D.P. and Naumann, V.O. (1959) 'Calculation of relative permeability from displacement experiments', Trans. AIME, Vol. 216, pp.370-372.

Josh, M., Esteban, L., Delle Piane, C., Sarout, J., Dewhurst, D.N. and Clennell, M.B. (2012) 'Laboratory characterisation of shale properties', J. Petrol. Sci. Eng., Vols. 88-89, pp.107-124.

Kanita, T., Forest, S., Gallieta, I., Mounourya, V. and Jeulina, D. (2003) 'Determination of the size of the representative volume element for random composites: statistical and numerical approach', Int. J. Solids Struct., Vol. 40, Nos. 13-14, pp.3647-3679.

Loeve, D., Wilschut, F., Hanea, R.H., Maas, J.G., van Hooff, P.M.O., van den Hoek, P.J., Douma, S.G. and Van Doren, J.F.M. (2011) 'Simultaneous determination of relative permeability and capillary pressure curves by assisted history matching several SCAL experiments', SCA Paper A46 prepared for International Symposium of the Society of Core Analysts, Austin, Texas, USA, September 2011.

Loomis, A.G. and Crowell, D.C. (1962) 'Relative permeability studies: gasoil and water-oil systems', USBM Bulletin, Vol. 599.

Matthï, S.K., Geiger, S., Roberts, S.G., Paluszny, A., Belayneh, M., Burri, A., Mezentsev, A., Lu, H., Coumou, D., Driesner, T. and Heinrich, C.A. (2007) 'Numerical simulation of multiphase fluid flow in structurally complex reservoirs', Geological Society London Special Publications, Vol. 292, No. 1, pp.405-429. 
Nguyen, V.H., Sheppard, A.P., Knackstedt, M.A. and Val Pinczewski, W. (2006) 'The effect of displacement rate on imbibition relative permeability and residual saturation', J. Petrol. Sci. Eng., Vol. 52, Nos. 1-4, pp.54-70.

Nichols, B.D. and Hirt, C.W. (1975) 'Methods for calculating multi-dimensional, transient free surface flows past bodies', Paper prepared for First Intern. Conf. Num. Ship Hydrodynamics, Gaithersburg, ML, October 1975.

Nichols, B.D. and Hirt, C.W. (1981) 'Volume of fluid (VOF) method for the dynamics of free boundaries', J. Comp. Phys., Vol. 39, No. 1, pp.201-225.

OpenFoam User Guide [online] http://www.openfoam.com/ (accessed 24 May 2013).

Papafotiou, A., Helmig, R., Schaap, J. and Lehman, P. (2008) 'From the pore scale to the lab scale: 3-D lab experiment and numerical simulation of drainage in heterogeneous porous media', Advances in Water Resources, Vol. 31, No. 9, pp.1253-1268.

Piller, M., Schena, G., Nolich, M., Favretto, S., Radaelli, F. and Rossi, E. (2009) 'Analysis of hydraulic permeability in porous media: from high resolution X-ray tomography to direct numerical simulation', Transport in Porous Media, Vol. 80, No. 1, pp.57-78.

Prodanović, M., Holder, J.T. and Bryant, S.L. (2012) 'Pore scale coupling of fluid displacement and unconsolidated sediment mechanics', International Journal of Oil, Gas and Coal Technology, Vol. 5, Nos. 2-3, pp.157-181.

ScanIP ${ }^{\circledR}$ by Simpleware [online] http://www.simpleware.com/software/scanip/ (accessed 29 May 2013).

Schena, G. and Favretto, S. (2007) 'Pore space network characterization with sub-voxel definition', Transport in Porous Media, Vol. 70, No. 2, pp.181-190.

Succi, S. (2001) The Lattice Boltzmann Equation for Fluid Dynamics and Beyond, Oxford University Press, Oxford, UK.

Tang, H., Wrobel, L.C. and Fan, Z. (2004) 'Tracking of immiscible interfaces in multiple-material mixing processes', Comput. Mater. Science, Vol. 29, No. 1, pp.103-118.

Tavassoli, Z., Carter, J.N. and King, P.R. (2005) 'Analysis of history matching errors', Computational Geosciences, Vol. 9, Nos. 2-3, pp.99-123.

Tiab, D. and Donaldson, E.C. (2004) Petrophysics: Theory and Practice of Measuring Reservoir Rock and Fluid Transport Properties, Elsevier Publications, Oxford, UK.

Toth, J., Bodi, T., Szucs, P. and Civan, F. (2002) 'Convenient formulae for determination of relative permeability from unsteady-state fluid displacements in core plugs', J. Petrol. Sci. Eng., Vol. 36, Nos. 1-2, pp.33-34.

Wolfram, S. (1986) 'Cellular automaton fluids 1: basic theory', Journal of Statistical Physics, Vol. 45, Nos. 3-4, pp.471-526.

Zaretskiy, Y., Geiger, S. and Sorbie, K. (2012) 'Direct numerical simulation of pore-scale reactive transport: applications to wettability alteration during two-phase flow', International Journal of Oil, Gas and Coal Technology, Vol. 5, Nos. 2-3, pp.142-156. 